\title{
A Comparative Evaluation of Mycobacterium w Vaccine Based Immunotherapy plus Concomitant Chemoradiation versus Concomitant Chemoradiation alone in Locally Advanced Head and Neck Cancer
}

\author{
Om Parkash ${ }^{1}$, Rakesh Dhankhar ${ }^{1}$, Kiran Dahiya ${ }^{2 *}$, Manas Dubey $^{1}$, Rajeev Atri ${ }^{1}$, Vivek Kaushal $^{1}$ \\ ${ }^{I}$ Department of Radiotherapy, Regional Cancer Centre, Pt. BD Sharma Postgraduate Institute of Medical \\ Sciences, Rohtak, Haryana, India \\ ${ }^{2}$ Department of Biochemistry, Pt. BD Sharma Postgraduate Institute of Medical Sciences, Rohtak, Haryana, \\ India
}

*Corresponding Author: Dr. Kiran Dahiya, Department of Biochemistry, Pt. BD Sharma Postgraduate Institute of Medical Sciences, 778/28, Bharat Colony, Rohtak, Haryana, PIN: 124001, India, Email:Kirandahiya_2002@yahoo.com

\begin{abstract}
Background: To compare the locoregional control and toxicity of Mycobacterium $w$ (Mw) vaccine based immunotherapy plus concomitant chemoradiation (MwCCRT) and concomitant chemoradiation (CCRT) alone in the patients of locally advanced head and neck cancer (LAHNC).

Materials and Methods: Sixty LAHNC patients were randomly assigned to either radical radiotherapy, 64 Gy/32 fractions over 6.2 weeks plus intravenous cisplatin $40 \mathrm{mg} / \mathrm{m}^{2}$ weekly for six doses (CCRT arm) or intradermal Mw vaccine $0.2 \mathrm{ml}$ weekly for six doses plus CCRT (MwCCRT arm) to equal number of patients. Primary end points taken were in the form of the response rate along with toxicity profile and locoregional control after 6 month follow up in two groups.
\end{abstract}

Results: All patients in each arm completed the planned treatment. The complete response rate was observed to be $70 \%$ versus $63.3 \%$ ( $p=0.752)$, no evidence of disease (NED) at six month of follow was seen in 63.3\% versus $53.3 \%$ in MwCRT and CRT arms $(p=0.612)$ respectively. Grade 2 skin and mucosal toxicity observed were $40 \%$ versus $83.3 \%(p=0.033)$ and $40 \%$ versus $83.3 \%(p=0.033)$ in MwCCRT arm and CCRT arm respectively. Grade 3 skin and mucosal toxicity were $13.3 \%$ and $10 \%$ in CCRT arm only, respectively.

Conclusion: This study on Indian locally advanced head and neck squamous cell carcinoma patients suggests that the use of weekly Mw vaccine plus weekly cisplatin is safe and MwCCRT may be superior to CCRT alone in terms of better response rate, higher locoregional control with lesser toxicities.

Keywords: Concomitant chemoradiotherapy, immunotherapy, locally advanced head and neck cancer, Mycobacterium $w(M w)$ vaccine based immunotherapy, squamous cell carcinoma

\section{INTRODUCTION}

Head and neck cancer (HNC) including lip, oral cavity, nasopharynx, other pharynx and larynx is the $7^{\text {th }}$ most common cancer globally. The incidence wise male-female ratio for $\mathrm{HNC}$ is nearly 3.7: 1 in India and nearly 2.7:1 globally [1].An increase in incidence of HNC (squamous cell carcinoma) is observed with increase in age and usually presents after 40 years of age [2]. Most of the head and neck cancers are triggered by alcohol and tobacco, which together probably account for three-quarters of cases [3].Tobacco smoking is associated with increased risk for all of the more common forms of $\mathrm{HNC}$, the risk among cigarette smokers may be ten or more times than that for non-smokers $[4,5]$.

Squamouscell carcinoma or a variant is the histology type in about $90 \%$ of HNC tumors [6]. $\mathrm{HNC}$ in the form of locally advanced disease is diagnosed in approximately $70-80 \%$ of these patients along with 30-50\% involvement of lymph nodes [7].Concomitant chemoradiotherapy (CCRT) is the standard treatment for patients with unresectable, non-metastatic locoregionally advanced squamous cell carcinoma of the head and neck (LASCCHN). Cisplatin based 
CCRT produces complete response in $80.5 \%$, Grade III and IV toxicities in $40 \%$, and threeyear overall survival in $62 \%$ patients $[8,9]$.

Immunotherapy for prevention and treatment of malignant diseases is in its initial stage. Initial work for urinary bladder cancer, melanoma and lung cancer has raised a hope that improving immune mechanism can be of use [10]. Randomized controlled studies in different cancers resulting in significant increase in survival and disease free period have been reported and its efficacy is enhanced by $20-30 \%$ when cell-based immunotherapy is combined with other conventional treatment methods [10].Bacillus Calmette-Guerin (BCG) immunetherapy for early stage (non-invasive) bladder cancer utilizes instillation of attenuated live bacteria into the bladder and is reported to be effective in preventing recurrence in up to two thirds of cases [11].

In few small clinical studies, Mycobacterium indicus pranii / Mycobacterium w (MIP / Mw) had been used in lung cancer and bladder cancer patients indicating its beneficial effect in the management of these diseases. The immunotherapeutic potential of $\mathrm{MIP} / \mathrm{Mw}$ in mouse tumor model was observed with higher number of immune cells $\left[\mathrm{CD} 4^{+}\right.$and $\mathrm{CD}^{+} \mathrm{T}$ cells, natural killer T (NKT) cells, macrophages and dendritic cells] infiltration in tumor cell mass in the MIP treated group as compared to control. These immune cells were in functionally active state in the MIP treated group with higher induction of proinflammatory Th1 type cytokines (like interferon gamma and interleukin-2) and increased cytotoxicity towards target tumor cells as compared to control group. The results in terms of delayed tumor growth and significantly less tumor volume were found in MIP treated mice tumor mass as compared to control tumor bearing mice [12].

The mainstay treatment modalities for LAHN Care radiotherapy and/or chemotherapy. The radiotherapy and/or chemotherapy cause acute toxicities which give rise to severe compliance problems [13,14].The use of Mycobacterium w (Mw) vaccine in $\mathrm{HNC}$ is an effort to reduce treatment induced toxicity with resultant improvement in the response rate.

Immunotherapy in the form of Mw vaccine may be useful as concurrent therapy with radical intent concomitant chemoradiation treatment to improve response rate with less side effects in patients suffering from locally advanced head and neck squamous cell carcinoma [15-18].

\section{MATERIALS AND METHODS}

\subsection{Study Design}

A prospective randomized single-center parallel comparative study was conducted at Department of Radiotherapy, Regional Cancer Centre, Pt B D Sharma Post-Graduate Institute of Medical Science, Rohtak. Institutional Board of postgraduate studies approved the protocol for the study.

\subsection{Patient Population}

Sixty previously untreated histopathologically proven patients of LAHNC (stage III-IV as per American Joint Committee on Cancer 7th edition, 2010) were enrolled for the study from March 2013 to September 2014, attending the Department of Radiotherapy for definitive treatment after obtaining informed written consent. The patients were randomly allocated to the study by draw of lots, 30 in Mw vaccine based immunotherapy plus concomitant chemoradiation arm (MwCCRT arm) and 30 in CCRT alone arm (CCRT arm). Inclusion criteria included Karnofsky performance status (KPS) > 70 and normal hematological, renal, and liver function tests. The patients having any of the following conditions were excluded from the study: distant metastases, prior treatment as radiation, surgery or chemotherapy for the disease, histopathology other than squamous cell carcinoma, pregnant or lactating females, co-morbid chronic/ endocrinal disease or any other malignancy and a poor general condition with Karnofsky Performance Status of $<70$. Thorough detailed clinical examination, history and workup details of all the patients were recorded.

\subsection{Treatment}

CCRT arm: concurrent chemoradiation intravenous (IV) cisplatin (DDP) 40 $\mathrm{mg} / \mathrm{m}^{2}$ weekly for six doses (i.e. days $1,8,15$, 22,29 , and 36) beginning from day 1 of radiation treatment (RT) and radical RT 64 Gy in 32 fractions over 6.2 weeks.

MwCCRT arm: intradermal (I/D) $0.2 \mathrm{ml}$ Mycobacterium w (Mw) vaccine based immunotherapy injection weekly for six doses (i.e. days $1,8,15,22,29$, and 36) beginning from day 1 of RT plus concomitant chemoradiation as in CCRT arm. Mw vaccine 
injections were given in bilateral deltoid regions.

Antiemetic regimen (injection dexamethasone $16 \mathrm{mg} \mathrm{IV}$, injection palonosetron $0.25 \mathrm{mg} \mathrm{IV}$, injection phenaramine maleate $25 \mathrm{mg}$ IV and injection ranitidine $50 \mathrm{mg}$ IV) was used 30 minutes before cisplatin administration and followed by prechemotherapy hydration with forced diuresis using mannitol 20\% IV. Injection cisplatin $40 \mathrm{mg} / \mathrm{m}^{2}$ IV was administered in $500 \mathrm{~mL}$ normal saline over one hour in both arms. Post-chemotherapyhydration was given immediately after the completion of cisplatin administration. Tablet ondansetron 8 $\mathrm{mg}$ and tablet ranitidine $150 \mathrm{mg}$ were given to patient per orally (PO) 12 hourly for next 5 days.

In both arms, the external beam radiotherapy was delivered to face and neck by conventional two parallel-opposed lateral fields in supine position on Cobalt-60 Teletherapy machine. Total dose of 64 Gy in 32 fractions over 6.2 weeks, 2 Gy per fraction, one fraction per day and 5 fractions per week was admintered to the mid plane at the central axis. Radiation treatment technique was individualized according to the site and extent of the disease.

Table1. Baseline characteristics of the patients
Spinal cord was spared from the radiation field after a dose of 44 Gy by using shrinking field technique. The patients were weekly assessed for treatment toxicities using World Health Organization (WHO) toxicity criteria. Patients who developed significant mucositis resulting in decreased oral intake were given IV alimentation along with Ryle's tube feeding. Patients were hospitalized to monitor any clinically significant toxicity. Complete blood counts, serum aspartate transaminase (AST), serum alanine transaminase (ALT), blood urea and serum creatinine were done every week. On treatment completion, the response was assessed and monthly follow up was carried out for six months using WHO response criteria. All patients underwent regular general physical, local and systemic examination every month for 6 months.

Statistical analysis was done applying Chisquare test.

\section{RESULTS}

The baseline characteristics of patients including demographic profiles, stages etc. are given in table1.

\begin{tabular}{|c|c|c|c|c|}
\hline \multicolumn{2}{|c|}{ Patients } & CCRT arm, n (\%) & MwCCRT arm, n (\%) & p value \\
\hline \multicolumn{2}{|l|}{ Number } & $30(100)$ & $30(100)$ & \begin{tabular}{|l|l|}
--- \\
\end{tabular} \\
\hline \multicolumn{2}{|l|}{ Males } & $28(93)$ & $27(90)$ & 0.321 \\
\hline \multicolumn{2}{|l|}{ Smokers } & $29(96)$ & $27(90)$ & 0.789 \\
\hline \multicolumn{2}{|l|}{ Alcoholics } & $20(67)$ & $18(60)$ & 0.209 \\
\hline \multirow{2}{*}{ KPS Score } & 80 & $22(73)$ & $23(77)$ & 0.881 \\
\hline & 90 & $08(27)$ & $07(23)$ & 0.231 \\
\hline \multirow{3}{*}{ Site of tumor } & Oropharynx & $19(63)$ & $21(70)$ & 0.752 \\
\hline & Hypopharynx & $05(17)$ & $02(7)$ & 0.732 \\
\hline & Larynx & $06(20)$ & $07(23)$ & 0.934 \\
\hline \multirow[b]{3}{*}{ Tumor size } & $\mathrm{T} 2$ & $08(27)$ & $04(13)$ & 0.142 \\
\hline & T3 & $21(70)$ & $20(67)$ & 0.087 \\
\hline & T4 & $01(03)$ & $06(20)$ & 0.321 \\
\hline \multirow[b]{2}{*}{ Nodal status } & Negative & $11(37)$ & $09(30)$ & 0.654 \\
\hline & Positive & $19(63)$ & $21(70)$ & 0.124 \\
\hline \multirow{2}{*}{ Stage } & III & $20(67)$ & $18(60)$ & 0.567 \\
\hline & IV & $10(33)$ & $12(40)$ & 0.241 \\
\hline
\end{tabular}

Both the groups were comparable with regard to age, sex, and clinical stage $(p>0.05)$. The median age at presentation was 58.7 years $(40-$ 85 years) in MwCCRT arm and 54.5 years (37-80 years) in CCRT arm respectively. The majority of patients $(69 \%)$ were older than 50 years in both the groups. The male to female ratio was 11:1. All efficacy analyses were done on an intent-to-treat basis. All patients had stage III or IV cancer at the time of presentation.

Haematological toxicity, gastrointestinal toxicity (nausea/vomiting), liver toxicity, renal toxicity, acute pharyngeal \& esophageal reactions (dysplasia), salivary gland reactions were detected more in CCRT arm than in 
A Comparative Evaluation of Mycobacterium w Vaccine Based Immunotherapy plus Concomitant Chemoradiation versus Concomitant Chemoradiation alone in Locally Advanced Head and Neck Cancer

MwCCRT arm though the difference was statistically not significant $(\mathrm{p}>0.05)$. Acute mucosal reactions and acute skin reactions are tabulated in table2 and table3 as per the

Table2. Mucosal reactions (MR) observed during treatment

\begin{tabular}{|c|c|c|c|c|c|c|c|c|}
\hline \multirow[b]{2}{*}{ Arm } & \multirow[b]{2}{*}{$\begin{array}{l}\text { RTOG } \\
\text { Grade }\end{array}$} & \multicolumn{7}{|c|}{ Number of patients } \\
\hline & & $\begin{array}{c}1^{\text {st }} \text { week } \\
\mathrm{n}(\%)\end{array}$ & $\begin{array}{c}2^{\text {nd }} \text { week } \\
\text { n }(\%)\end{array}$ & $\begin{array}{c}3^{\text {rd }} \text { week } \\
\text { n }(\%)\end{array}$ & $\begin{array}{c}4^{\text {th }} \text { week } \\
n(\%)\end{array}$ & $\begin{array}{c}5^{\text {th }} \text { week } \\
n(\%)\end{array}$ & $\begin{array}{c}6^{\text {th }} \text { week } \\
\text { n }(\%)\end{array}$ & $\begin{array}{c}\text { Completion } \\
\mathrm{n}(\%)\end{array}$ \\
\hline \multirow{5}{*}{$\begin{array}{l}\text { MwCCRT } \\
n=30\end{array}$} & 0 & $30(100)$ & $28(93$ & $19(63)$ & $07(23)$ & 0 & 0 & 0 \\
\hline & 1 & 0 & $02(07)$ & $10(33)$ & $21(70)$ & $24(80)$ & $19(63)$ & $18(60)$ \\
\hline & 2 & 0 & 0 & $01(03)$ & $02(07)$ & $06(20)$ & $11(36)$ & $12(40)$ \\
\hline & 3 & 0 & 0 & 0 & 0 & 0 & 0 & 0 \\
\hline & 4 & 0 & 0 & 0 & 0 & 0 & 0 & 0 \\
\hline \multirow{5}{*}{$\begin{array}{l}\text { CCRT } \\
n=30\end{array}$} & 0 & $30(100)$ & $22(73)$ & $14(47)$ & $04(13)$ & 0 & 0 & 0 \\
\hline & 1 & 0 & $08(27)$ & $13(43)$ & $19(63)$ & $08(27)$ & $03(10)$ & $02(07)$ \\
\hline & 2 & 0 & 0 & $03(10)$ & $07(23)$ & $22(73)$ & $24(80)$ & $25(83)$ \\
\hline & 3 & 0 & 0 & 0 & 0 & 0 & $03(10)$ & $03(10)$ \\
\hline & 4 & 0 & 0 & 0 & 0 & 0 & 0 & 0 \\
\hline
\end{tabular}

Grade 2 MR at the $4^{\text {th }}$ week of treatment: Chi square $2.77, p=0.96$

Grade 2 MR at the $5^{\text {th }}$ week of treatment: Chi square 9.143, $p=0.002$

Grade 2 MR at the $6^{\text {th }}$ week of treatment: Chi square $4.829, p=0.028$

Grade $2 \mathrm{MR}$ at the end of treatment: Chi square 4.568, $p=0.033$

For grade $3 \mathrm{MR}$, group I is constant

Table3.Skin reactions observed during treatment

\begin{tabular}{|c|c|c|c|c|c|c|c|c|}
\hline \multirow[b]{2}{*}{ Arm } & \multirow[b]{2}{*}{$\begin{array}{l}\text { RTOG } \\
\text { Grade }\end{array}$} & \multicolumn{7}{|c|}{ Number of patients } \\
\hline & & $\begin{array}{l}1^{\mathrm{st}} \mathrm{wk}, \\
\mathrm{n}(\%)\end{array}$ & $\begin{array}{c}2^{\text {nd }} w k, \\
n(\%)\end{array}$ & $\begin{array}{l}3^{\text {rd }} \text { wk, } \\
\mathrm{n}(\%)\end{array}$ & $\begin{array}{c}4^{\text {th }} \mathrm{wk}, \\
\mathrm{n}(\%)\end{array}$ & $\begin{array}{c}5^{\text {th }} w \mathrm{k}, \\
\mathrm{n}(\%)\end{array}$ & $\begin{array}{l}6^{\text {th }} \mathrm{wk}, \\
\mathrm{n}(\%)\end{array}$ & $\begin{array}{c}\text { Completion } \\
\mathrm{n}(\%)\end{array}$ \\
\hline \multirow{5}{*}{$\begin{array}{l}\text { MwCCRT } \\
n=30\end{array}$} & 0 & $30(100)$ & $28(93)$ & $20(67)$ & $02(07)$ & 0 & 0 & 0 \\
\hline & 1 & 0 & $02(07)$ & $10(33)$ & $27(90)$ & $20(67)$ & $18(60)$ & $17(57)$ \\
\hline & 2 & 0 & 0 & 0 & $01(03)$ & $10(03)$ & $12(40)$ & $13(43)$ \\
\hline & 3 & 0 & 0 & 0 & 0 & 0 & 0 & 0 \\
\hline & 4 & 0 & 0 & 0 & 0 & 0 & 0 & 0 \\
\hline \multirow{5}{*}{ CCRT } & 0 & $30(100)$ & $25(83)$ & $13(43)$ & $03(10)$ & 0 & 0 & 0 \\
\hline & 1 & 0 & $05(17)$ & $14(47)$ & $18(60)$ & $08(27)$ & $01(03)$ & $01(03)$ \\
\hline & 2 & 0 & 0 & $03(10)$ & $09(30)$ & $22(73)$ & $25(83)$ & $25(83)$ \\
\hline & 3 & 0 & 0 & 0 & 0 & 0 & $04(13)$ & $04(13)$ \\
\hline & 4 & 0 & 0 & 0 & 0 & 0 & 0 & 0 \\
\hline
\end{tabular}

Grade II skin reaction at the $4^{\text {th }}$ week of treatment: Chi square 6.40, $p=0.011$

Grade II skin reaction at the $5^{\text {th }}$ week of treatment: Chi square 4.5, $p=0.034$

Grade II skin reaction at the $6^{\text {th }}$ week of treatment: Chi square 4.568, $p=0.033$

Grade II skin reaction at the end of treatment: Chi square 3.789, $p=0.052$

For grade III skin reaction, group I is constant

Grade 3 mucosal reactions and Grade 3 skin reactions were detected only in $10 \%$ in MwCCRT and $13 \%$ patients of CCRT arm respectively. Higher mucosal and skin toxicities were found in CCRT arm and the difference in two arms was statistically significant for grade 2 reactions $(\mathrm{p}<0.05)$. The local abscesses at intradermal $\mathrm{Mw}$ vaccine injection sites (deltoid region of arm) were detected in seven patients $(23.3 \%)$ in MwCCRT arm only on weekly observation during the treatment. Table 4 shows the complete response (CR) and partial response (PR) at primary site and at nodal site individually. Table 5 shows the response at primary site and nodal site together. CR was observed to be better in MwCCRT arm than CCRT arm though not significant statistically 
$(\mathrm{p}=0.612$ ). Six months of follow-up (range 618months) was completed by all patients and the observations are tabulated in Table 6 . The local tumor control was better found in MwCCRT arm as compared to CCRT arm though not significant statistically $(\mathrm{p}=0.612)$.

Table4. Response at primary and nodal sites individually (WHO response criteria)

\begin{tabular}{|l|l|l|l|l|}
\hline \multicolumn{2}{|c|}{ WHO response criteria } & \multicolumn{1}{|c|}{ MwCCRT arm } & \multicolumn{1}{c|}{ CCRT arm } & \multicolumn{1}{c|}{ p value } \\
\hline $\begin{array}{l}\text { Response at } \\
\text { primary site }\end{array}$ & CR & $21 / 30(70)$ & $19 / 30(63)$ & 0.612 \\
\cline { 2 - 5 } $\begin{array}{l}\text { Response at } \\
\text { nodal site }\end{array}$ & PR & $09 / 30(30)$ & $11 / 30(37)$ & \\
\cline { 2 - 5 } & PR & $15 / 21(71)$ & $12 / 19(63)$ & 0.275 \\
\hline
\end{tabular}

Figures indicated in parenthesis are percentage

Abbreviation: CR- complete response, PR-partial response

Table5. Response at primary and nodal site together (WHO response criteria)

\begin{tabular}{|l|l|l|c|}
\hline \multicolumn{1}{|c|}{ WHO response criteria } & \multicolumn{1}{c|}{ MwCCRT arm } & \multicolumn{1}{c|}{ CCRT arm } & \multicolumn{1}{c|}{ p value } \\
\hline CR & $21 / 30(70.0)$ & $19 / 30(63.33)$ & 0.612 \\
\hline PR & $09 / 30(30.0)$ & $11 / 30(36.66)$ & \\
\hline
\end{tabular}

Figures indicated in parenthesis are percentage

Abbreviation: CR-complete response, PR-partial response

Table6. Disease status at six months of follow-up

\begin{tabular}{|l|l|l|l|l|}
\hline \multirow{2}{*}{\multicolumn{1}{c|}{ Arm }} & \multirow{2}{*}{$\begin{array}{c}\text { Total no. of } \\
\text { patients }\end{array}$} & \multicolumn{3}{c|}{ Disease Status } \\
\cline { 3 - 6 } & 30 & NED & RD & \multicolumn{1}{c|}{ REC } \\
\hline MwCCRT & 30 & $19(63.3 \%)$ & $09(30.0 \%)$ & $02(06.7 \%)$ \\
\hline CCRT & 30 & $16(53.3 \%)$ & $10(33.3 \%)$ & $04(13.3 \%)$ \\
\hline
\end{tabular}

$N E D=$ No evidence of disease, $R D=$ Residual disease, $R E C=$ Recurrent disease

Chi square 0.257, $p=0.612$ for NED status in all stages

\section{DISCUSSION}

The treatment modalities in advanced stage of HNC are radiotherapy and/or chemotherapy which might be associated with acute toxicities. The present study using Mw vaccine immunotherapy along with CCRT in the management of LASCCHN has demonstrated the reduction in treatment induced toxicity with improvement in clinical response. Similar results have been reported by Dave et al who conducted a randomized controlled study to evaluate the effect of vaccine containing $\mathrm{Mw}$ administered for 6 months in 100 patients of head and neck cancer patients and concluded that immunotherapy may be useful as a safe therapy in head and neck cancer patients for better clinical response, reducing side effects and improving quality of life [18].

Another study carried out by Sarkar et alhas alsoreported the similar findings. This research work was conducted using $0.1 \mathrm{ml}$ Mycobacterium $w$ vaccine given intracutaneously weekly during radiotherapy (6000-6600 cGy/6-61/2 weeks) along with cisplatin $30 \mathrm{mg} / \mathrm{m}^{2}$ weekly for 6 weeks and continued every 2 weeks for another 3 months after completion of radiation schedule in study arm of 30 patients with squamous cell carcinoma of head and neck. The patients included in the control arm were 30 evenly matched head and neck cancer patients and treated by the same schedule but without any adjuvant immunomodulator. They observed no grade III skin or hematological toxicity and no interruption in treatment protocol in the study arm whereas grade III skin/mucous membrane/hematological toxicities leading to interruption of treatment schedule and much prolonged treatment time was found in 12 out of 30 patients in the control arm. There was no statistically significant difference between the two treatment arms regarding disease response. Quality of life as measured by Karnofsky Performance Status was significantly better in the study arm. They concluded that Mycobacterium $w$ is an economically viable and well tolerated immunomodulator for head and neck cancer patients, producing significant reduction in treatment related acute toxicities and producing enhanced radiation tolerance [16].

The above trials are in accordance with the present study and have reported better locoregional control (tumor clinical response) along with reduced treatment induced toxicity 
in the $\mathrm{Mw}$ vaccine based immunotherapy groups along with CCRT (MwCCRT group) as compared to the CCRT alone groups (control group) [16,18].

Immunotherapy, also called biologic therapy, is a type of cancer treatment that boosts the body's natural defenses to fight cancer. Several immunosuppressive immune subsets have been found in HNC including tumour associated macrophages, myeloid-derived suppressor cells, and regulatory $\mathrm{B}$ and $\mathrm{T}$ cells. Induction of anti-tumor immunity is a multistep process, which is regulated at every step by positive and negative signals. The tumor antigens first reach professional antigenpresenting cells (APCs), such as dendritic cells (DCs), which upon activation migrate to draining lymph nodes. There, DCs, after processing the peptides in the proteasome, present them as part of the major histocompatibility (MHC) molecules to T cells resulting in $\mathrm{T}$ cell activation and proliferation. Upon activation, tumor-specific $\mathrm{T}$ cells differentiate into effect or cells or memory $\mathrm{T}$ cells, which carry out different anti-tumor activities. Immunotherapies including mycobacterium $\mathrm{w}$ vaccine act at one or more of the steps of this process and may prove to be need of the day in showing better tumor control as well as being less toxic in LASCCHNC in concert with CCRT $[19,20]$.

\section{CONCLUSION}

The observations made in the study suggest that $\mathrm{Mw}$ vaccine based immunotherapy along with CCRT might prove to be a better approach for treatment of patients with LASCCHN as compared to CCRT alone.

\section{REFERENCES}

[1] Tuljapurkar V., Dhar H., Mishr A., Chakraborti S., Chaturvedi P. and Pai P. S., The Indian scenario of head and neck oncology: challenging the dogmas, South. Asian. J. Cancer. 5(3), 105-110 (2016).

[2] Ma X.and Yu H., Global Burden of Cancer, Yale. J. Biol. Med. 79(3-4), 85-94 (2006).

[3] Poddar A., Aranha R. R., K Muthukaliannan G., Nachimuthu R. and Jayaraj R.,Head and neck cancer risk factors in India: protocol for systematic review and meta-analysis, B. M. J. Open. 8(8), e020014 (2018).

[4] Wu S., Zhu W., Thompson P. and Hannun Y. A., Evaluating intrinsic and non-intrinsic cancer risk factors, Nat. Commun. 9(1), 3490 (2018).
[5] Nayan S., Gupta M. K. and Sommer D. D, Evaluating smoking cessation interventions and cessation rates in cancer patients: a systematic review and meta-analysis, ISRN Oncol. 2011, 849023 (2011).

[6] Dhankhar R., Dahiya K., Kumar R., Atri R., Chugh K., Kamboj K and Singh S., How Lipid Profile Gets Affected in Head And Neck Squamous Cell Carcinoma Before And After Treatment?, Clin. Oncol. 1(1), 1-5 (2018).

[7] Manocha S., Suhag V., Sunita B. S., Hooda H. S. and Singh S., Comparison of sequential chemoradiation with radiation alone in the treatment of advanced head and neck cancers, Indian. J. Otolaryngol. Head. Neck. Surg. 58(1), 57-60 (2006).

[8] Sharma A., Mohanti B. K., Thakar A., Bahadur S. and Bhasker S., Concomitant chemoradiation versus radical radiotherapy in advanced squamous cell carcinoma of oropharynx and nasopharynx using weekly cisplatin,Ann. Oncol. 21(11), 2272-2277 (2010).

[9] Hitt R., Grau J. J., López-Pousa, Berrocal A., García-Girón C., Irigoyen A., Sastre J., Martínez-Trufero J., Brandariz Castelo J. A., Verger E. and Cruz-Hernández J. J., Spanish Head and Neck Cancer Cooperative Group (TTCC). A randomized phase III trial comparing induction chemotherapy followed by chemoradiotherapy versus chemoradiotherapy alone as treatment of unresectable head and neck cancer, Ann. Oncol. 25(1), 216-225 (2014).

[10] Chhatui B., Devleena., Roy S., Maji T., Lahiri D. and Biswas J., Immunomodulated anterior chemotherapy followed by concurrent chemoradiotherapy in locally advanced tongue cancer: An Institutional experience, Indian. J. Med. Paediatr. Oncol. 36(1), 43-48 (2015).

[11] Sylvester R. J., van der MEIJDEN A. P. andLamm D.L.,Intravesical Bacillus Calmette-Guerin reduces the risk of progression in patients with superficial bladder cancer: a meta-analysis of the published results of randomized clinical trials,J. Urol. 168(5), 1964-1970 (2002).

[12] Ahmad F., Mani J., Kumar P., Haridas S., Upadhyay P. and Bhaskar S., Activation of anti-tumor immune response and reduction of regulatory $\mathrm{T}$ cells with Mycobacterium indicus pranii (MIP) therapy in tumor bearing mice, PLoS. One. 6(9), e25424 (2011).

[13] Pitman K. T. and Dean R., Management of the clinically negative (N0) neck, Curr. Oncol. Rep. 4(1), 81-6 (2002).

[14] Majumder D., Choudhary K., Das P., Kundu S. and Mitra D., Different fractionation 
schedules of radiotherapy in locally advanced head and neck malignancy: A prospective randomized study to compare the results of treatment and toxicities of different protocols. South. Asian. J. Cancer. 2(1), 31-35 (2013).

[15] Guidi A., Codecà C. and Ferrari D., Chemotherapy and immunotherapy for recurrent and metastatic head and neck cancer: a systematic review, Med. Oncol. 35(3), 37 (2018).

[16] Sarkar S.K. and Dasgupta C., Role of Mycobacterium $\mathrm{W}$ as an adjuvant treatment of Head \& Neck Cancer--A Randomised trial, J. Clin. Oncol. 23(16), 5588 (2005).

[17] Smith S. G., Zelmer A., Blitz R., Fletcher H. A. and Dockrell H. M., Polyfunctional CD4 T-cells correlate with in vitro mycobacterial growth inhibition following Mycobacterium bovis BCG-vaccination of infants, Vaccine. 34(44), 5298-5305 (2016).

[18] Dave R.I., Tripathi U. B., Parikh B. J., Patel K. M. and Shukla H. K., Improvement in quality of life and symptom control with MYCOBACTERIUM W (CADI-05) in head and neck cancer, J. Clin. Oncol. 25(18), 21186 (2007).

[19] Chen D. S. and Mellman I., Oncology meets immunology: the cancer-immunity cycle, Immunity. 39(1), 1-10 (2013).

[20] Parker J. J., Jones J.C., Strober S. and Knox S. J., Characterization of direct radiationinduced immune function and molecular signaling changes in an antigen presenting cell line, Clin. Immunol. 148(1), 44-55 (2013).

Citation: Om Parkash, Rakesh Dhankhar, Kiran Dahiya, Manas Dubey, Rajeev Atri, Vivek Kaushal, A Comparative Evaluation of Mycobacterium $w$ Vaccine Based Immunotherapy plus Concomitant Chemoradiation versus Concomitant Chemoradiation alone in Locally Advanced Head and Neck Cancer. ARC Journal of Cancer Science 2018; 4(2):18-24. DOI:dx.doi.org/10.20431/2455-6009.0402005.

Copyright: (C) 2018 Authors. This is an open-access article distributed under the terms of the Creative Commons Attribution License, which permits unrestricted use, distribution, and reproduction in any medium, provided the original author and source are credited. 